\title{
"El problema del agua no es solo el agua": reflexiones sobre el diálogo desde una experiencia educativa con poblaciones sin acceso al agua potable, en Turbo (Colombia) entre 2015 y 2016'
}

\section{"The problem of water is not only water": reflections about dialogue around an educational experience in communities without access to potable water, Turbo (Colombia) 2015-2016}

\section{Paulina Díaz-Mosquera ${ }^{a}$ \\ (iD) https://orcid.org/0000-0002-4885-6807 \\ E-mail: silvia.diazœudea.edu.co \\ Natalia Rodríguez-Villamil ${ }^{b}$ \\ (D) https://orcid.org/0000-0001-7237-1917 \\ E-mail: luz.rodriguezळudea.edu.c \\ Alejandra Valencia-González ${ }^{c}$ \\ (i) https://orcid.org/0000-0002-1517-2177 \\ E-mail: alejandra.valenciaœudea.edu.co}

anniversidad de Antioquia. Facultad de Odontología. Departamento de Estudios Básicos. Medellín, Colombia.

buniversidad de Antioquia. Escuela de Nutrición y Dietética. Departamento de Formación Académica. Medellín, Colombia.

'Universidad de Antioquia. Facultad de Enfermería. Departamento de Formación Básica Profesional. Medellín, Colombia.

\section{Resumen}

En este trabajo se presenta una reflexión sobre una experiencia educativa con comunidades ubicadas en el casco urbano del municipio de Turbo (Colombia), que no tienen acceso al agua potable. Los participantes son 18 líderes comunitarios, 149 familias de tres barrios y profesionales de dentro y fuera de la región. Este proceso tuvo como objetivo el fortalecimiento de las prácticas del uso cotidiano y conservación del agua en las comunidades, y es respuesta a una investigación previa que analizó algunos retos que enfrentan las personas para acceder a fuentes de agua suficientes y adecuadas para suplir sus necesidades. En esta experiencia se privilegia el diálogo como posibilidad para el reconocimiento de las condiciones de acceso al agua potable y la construcción de acciones para la gestión y defensa del derecho al agua.

Palabras clave: Diálogo; Educación para la Salud; Agua; Agua Potable; Conocimientos, Actitudes y Prácticas en Salud.

\section{Correspondencia}

Paulina Díaz-Mosquera

Calle 70 \# 52-21, Medellín, Antioquia, Colombia. Código Postal: 050010.

\footnotetext{
1 Este proyecto fue financiado por el Banco Universitario de Programas y Proyectos de Extensión (Buppe) de la Universidad de Antioquia, IX Convocatoria BUPPE de Proyectos de Convergencia Académica y Social 2014, código del proyecto: 3241141. Además, contó con la cofinanciación de Corpourabá para el diseño y elaboración de materiales educativos.
} 
This paper presents a reflection on an educational experience with communities located in the urban area of the municipality of Turbo (Colombia), which do not have access to potable water. The participants are eighteen community leaders, one hundred and forty-nine families from three neighborhoods and professionals from inside and outside the territory. The main objective was to strengthen the practices of daily use and conservation of water in the communities. This process is related to a previous research that analyzed the challenges faced by people to access to sufficient and adequate water sources to meet their needs, among others. In this experience, dialogue was the possibility to recognize the conditions to have access to potable water, and to define collective actions for the management and defense of the right to water.

Keywords: Dialogue; Health Education; Water; Water Potable; Health Knowledge, Attitudes, Practice.
Este escrito pretende reflexionar sobre el diálogo como eje movilizador de procesos educativos en salud, a partir de una experiencia vivida durante los años 2015-2016 con familias sin acceso al agua potable, habitantes de los barrios El Esfuerzo, El Pescador 1 y 2, ubicados en el casco urbano del municipio de Turbo, Antioquia (Colombia). Esta experiencia educativa tuvo por objetivo el fortalecimiento de sus prácticas de uso cotidiano y conservación del agua, y nació como respuesta a lo encontrado en una investigación previa realizada en el mismo contexto, que describió las percepciones, prácticas y usos relacionados con el agua y analizó los retos que enfrentan las personas para acceder a fuentes de agua suficientes y adecuadas, tratarla adecuadamente y enfrentar el desgaste corporal de niños y adultos para su transporte (Rodríguez; Restrepo; Zambrano, 2013). Uno de los retos metodológicos que enfrentamos en esta experiencia tuvo que ver con el establecimiento de un proceso educativo en el que se pudiera construir una interacción situada y pertinente con la participación de actores diversos, algunos de ellos en conflicto. Encontramos en el diálogo la mediación necesaria para hacerlo y, por ello, compartimos el camino transitado en este proyecto.

Concebir un proceso de educación para la salud (EpS) implica, de acuerdo con Valadez, Villaseñor y Alfaro (2004), conocer los modelos, concepciones, sentidos y discursos teóricoepistemológicos que dan forma al acto educativo. Para la relación educativa, centro de reflexión de este escrito, Ramasco (2014) clasifica cuatro modelos; dos de ellos centran la relación en el conocimiento del profesional: el informativo persuasivo y el motivacional, mientras que en el modelo políticoeconómico-ecológico el profesional se asume como mediador, y en el crítico-emancipatorio, se propone una cogestión del aprendizaje. Serrano (1990, 2002) propone un modelo para la EpS de participación y compromiso en la que acoge, entre otros, el diálogo como instrumento central del proceso, a partir de las situaciones de la vida para llegar a una "pregunta sanitaria" que permita analizar de manera crítica el contexto y analizar la EpS como posibilidad de 
aportar a comunidades que se conviertan en redes de comunicación saludable.

Para este proyecto, la EpS no se nombró desde algún modelo en particular, pero se construyó desde los siguientes elementos: la planeación a partir del conocimiento construido en la investigación previa; la voluntad de las comunidades de hacer parte de un proceso que mejorara su situación con el agua; la inclusión de personas e instituciones relacionadas con el agua en el municipio; la planeación de acciones didácticas que reconocieran las condiciones particulares de los participantes; la flexibilidad del proceso; y el reconocimiento del derecho al agua y a la salud en conexión con el derecho a una vida digna.

La gestión y mediación del proyecto estuvo a cargo de profesoras de la Escuela de Nutrición y Dietética y las facultades de Enfermería y de Odontología de la Universidad de Antioquia (Colombia), quienes partieron de sus experiencias relacionadas con la EpS desde el marco comprensivo de la salud colectiva, que considera "la vida y la salud como realidad cultural, ética y veraz, que se produce en el cotidiano vivir de la gente" (Granda, 2009, p. 107) y al sujeto como eje central de las acciones en salud.

Uno de los aprendizajes de este proceso fue el diálogo como mediador de las relaciones. Al respecto cabe mencionar, entre otros referentes, a Freire (1975), quien reflexiona sobre la dialogicidad de la educación como encuentro que solo es posible entre sujetos que pronuncian el mundo en sus dimensiones de acción y reflexión; y a Mariño (2016), quien analiza la importancia de reconocer diversos tipos de diálogo, de reflexionar y aprender sobre las maneras como este se lleva a cabo.

Existe también una amplia base de discusión sobre la importancia del diálogo de saberes y el diálogo cultural e intercultural como parte fundamental de los procesos educativos para la salud, en ámbitos individuales y comunitarios. Encontramos así el diálogo de saberes como eje de experiencias de educación para la salud, entendido por autores como Bastidas et al. (2009) como propuesta pedagógica que pone en interacción la lógica del conociminento científico y el saber cotidiano, con la intención de comprenderse mutuamente por medio del reconocimiento de las diferencias; y Briceño (1996), quien propone que el diálogo debe darse en condiciones de equidad, multidireccionalidad y negociación, fomentando la autonomía, el respeto y la transformación de quienes participan. Restrepo (2012) destaca la transdisciplinariedad como vía para materializar el diálogo de saberes, incluyendo saberes expertos y otros adquiridos a través de la experiencia social. Hernández et al. (2017), por su parte, acogen el diálogo de saberes como un método cualitativo en el marco de la investigación, acción participativa, que permite una aproximación intercultural y transdisciplinaria para comprender y contextualizar representaciones y prácticas sociales que permita entender los problemas y necesidades en salud de las personas.

Acerca del diálogo cultural, algunas reflexiones apuntan al reconocimiento de que "los saberes de los sujetos educativos están inmersos en matrices culturales y entramados simbólicos que deben ser comprendidos y afectados desde la acción pedagógica" (Ortega; Torres, 2011, p. 353), lo que invita a ampliarlo a los aspectos socioafectivos y culturales presentes en los sujetos que enuncian.

En cuanto al diálogo intercultural, Astaiza et al. (2012) reflexionan sobre las interacciones con comunidades indígenas o afro y plantean el diálogo desde una interacción cultural positiva, que tiene condiciones de mutuo enriquecimiento, empoderamiento y autonomía. Por su parte, Espinoza e Ysunza (2009) resaltan la apertura de espacios dialógicos para definir condiciones consensuadas de articulación de los conocimientos y las prácticas del saber hegemónico y otros saberes subalternos.

En estas perspectivas existe un punto común: el diálogo como espacio de acercamiento con otros a partir de la diferencia en la visión del mundo o el tipo de saber (científico/cotidiano, de expertos/de experiencia, hegemónico/subalterno), que transforma a quienes participan y aporta a la comprensión o la autonomía. En este artículo consideramos pertinente fortalecer la discusión sobre el diálogo sin adjetivaciones o caracterizaciones, rescatando lo común a todas, esto es, la importancia de lo dialógico en los procesos educativos para la salud, centrándonos en la manera en que acontece, como espacio que incluye acuerdos, pero también divergencias, conflictos y disensos propios de la experiencia humana. 
Así este artículo se desarrolla en tres apartados. En el primero recreamos el contexto de Turbo en el momento de ejecución del proceso, en el segundo resaltamos algunas experiencias y expresiones recogidas en la interacción con los participantes y finalizamos con algunos aprendizajes acerca del diálogo en la EpS.

\section{La dinámica del proceso}

Turbo es un municipio costero habitado por cerca de 160.00o habitantes, ubicado en la subregión de Urabá, en el departamento de Antioquia (Colombia). Su clima es cálido, lo que favorece una de sus principales actividades económicas: la producción y exportación de banano y plátano. La actividad pesquera es artesanal y de subsistencia (Alcaldía Municipal de Turbo, 2016; Congreso de Colombia, 2018). En el Urabá antioqueño, las cifras de pobreza superan ampliamente el promedio departamental. En cuanto al acceso al agua, mientras en Antioquia, para la época de ejecución del proyecto, la cobertura del servicio de acueducto alcanzaba el $88 \%$, en Urabá solo llegaba al 35\% (Gobernación de Antioquia, 2012). En Turbo, según el Plan de Desarrollo Municipal vigente para la época, el acueducto solo cubría el $45 \%$ del sector urbano y el $38 \%$ del rural; con cortes frecuentes y con agua no potable y escasa (Alcaldía Municipal de Turbo, 2012). Aunque existe planta de tratamiento, las conexiones informales realizadas en diversos barrios hacen que el agua que sale potable llegue a las viviendas contaminada con aguas subterráneas y residuales.

Ese mismo Plan de Desarrollo (Alcaldía Municipal de Turbo, 2012) señala que la falta de agua potable, junto con el desplazamiento forzado, la vivienda en malas condiciones y la contaminación por productos químicos, son causas de morbilidad en la población infantil y adulta. La situación más dramática la enfrentan los habitantes de barrios informales, marginados de la planeación municipal. En esta situación se encuentran los barrios El Esfuerzo, Pescador 1 y 2.

El Esfuerzo es un asentamiento conformado aproximadamente en 2010, en la zona aledaña a las lagunas de oxidación que reciben las aguas residuales del casco urbano. La población es mayoritariamente afrocolombiana, muchos de ellos llegaron al municipio por desplazamiento forzado. Según un censo realizado por la comunidad, en 2015 habitaban el sector aproximadamente 700 personas. Las viviendas y calles fueron construidas por los habitantes; existe servicio de energía eléctrica en modalidad prepago y el agua la compran en barrios vecinos, la toman de pozos artesanales, la lluvia o una quebrada cercana.

Los barrios Pescador 1 y 2 se establecieron hace más de 35 años; los censos comunitarios reportan que habitan allí respectivamente 700 y 2.500 personas, en unas 450 viviendas ubicadas cerca de la playa, en zona de manglares. Algunos de sus habitantes nacieron en Turbo, otros llegaron de diferentes municipios, en algunos casos por desplazamiento forzado. Las familias se abastecen de agua mediante motobombas, la compran envasada, la traen de otros barrios, la toman de la lluvia o de un carro tanque que llega en tiempo de sequía. Existen calles y viviendas en materiales permanentes, y en buena parte del sector hay palafitos y tablas sobre el manglar, a manera de vías.

La limitada disponibilidad de agua se explica por los aspectos ya mencionados a los que se suman: una escasa organización comunitaria, el bajo caudal del río que abastece al acueducto, un sistema de tuberías obsoleto, el uso de motobombas que descompensan el sistema y un alto nivel freático que dificulta la construcción de un nuevo acueducto; en últimas la situación se deriva del cambio climático global y la inadecuada relación que hemos tenido con la naturaleza, lo cual para estas comunidades se agrava ante sus condiciones de asentamiento y exclusión de la planeación municipal, hecho que las ha llevado a tener prácticas con el agua que no siempre son seguras para ellos ni para el ambiente. Por lo tanto, el golfo de Urabá se convierte en actor en pugna, porque representa un territorio cuyo cuidado entra en contradicción con el crecimiento poblacional sobre el manglar, lo que afecta la vida marina y, por ende, la de la población.

En este contexto fuimos recibidas con gran calidez por habitantes y representantes de instituciones; las condiciones climáticas de 2015, también cálidas, nos presentaron el reto de convocar a un proyecto para poblaciones sin acceso al agua potable, en la época de sequía más grande de los últimos años y en medio de un año de elección de alcaldes, gobernadores, asamblea y concejos municipales. 
Un punto de partida fue el reconocimiento permanente de que quienes movilizábamos el proceso no pertenecíamos al territorio, lo que hizo manifiesta nuestra condición de aprendices y la importancia de involucrar a otros como protagonistas, entre ellos los participantes de los barrios. Iniciamos un mapeo preliminar de actores que incluyó a organizaciones gubernamentales y no gubernamentales, empresas privadas, líderes de los barrios, participantes de la investigación previa, profesores, personal administrativo y estudiantes de la seccional Urabá de la Universidad de Antioquia. Este mapeo buscaba establecer alianzas y reconocer espacios, personas y relaciones, lo que condujo a una "bola de nieve" en la que encontramos otros actores pertinentes para el proceso. Lo anterior permitió reconfigurar la confianza ya construida desde el proceso de investigación e integrar los elementos del proyecto educativo.

Finalmente se vincularon diferentes actores que aportaron a los objetivos del proyecto desde diversas áreas del conocimiento: una institución ambiental que además cofinanció algunos materiales educativos, la Universidad de Antioquia, seccional Urabá con apoyo logístico e infraestructura, una confederación internacional que realiza trabajo humanitario, con la asesoría en la adquisición de filtros para potabilización de agua, una entidad de aseo municipal, con un taller relacionado con el cuidado de las fuentes de agua y la empresa de acueducto, con una visita guiada a la planta de tratamiento.

Además, según las necesidades de cada actividad, se integraron profesionales de diversas áreas: ecólogo de zonas costeras, administrador en salud con énfasis en gestión sanitaria y ambiental, ingeniera química, comunicadora social, educador físico, biólogo marino, abogados, especialista en gestión integral de residuos sólidos, diseñador gráfico, estudiantes de nutrición y dietética y de tecnología en saneamiento ambiental.

Se convocó a zo líderes de los barrios, que se reconocían del proceso investigativo previo, quienes firmaron un consentimiento para su participación, en el cual se enfatizaba en la confidencialidad de los datos personales y el respeto a las decisiones de los participantes respecto al proyecto. Aunque esta experiencia no constituye un proyecto de investigación, para ser aprobada por la Universidad de Antioquia fue revisada en todos sus aspectos, incluidos los éticos, por el comité que definía su viabilidad.

El proceso de formación fue completado por 18 líderes (11 mujeres y 7 hombres), el 61\% de ellos, afrodescendientes, y el 39\%, mestizos, quienes llevaban viviendo en sus barrios entre 3 y 40 años, se dedicaban principalmente a trabajos informales cuya escolaridad iba desde el analfabetismo hasta la secundaria.

El proceso educativo se realizó mediante talleres (Cuadro 1) sobre prácticas de manejo del agua y temas relacionados, en encuentros realizados en sus territorios y en espacios institucionales, documentados en un portafolio donde se consignaron, taller a taller, los aprendizajes a través de infografías adecuadas a sus posibilidades lectoescriturales. Así mismo, planearon y ejecutaron actividades para compartir lo aprendido con otros y respondieron algunas entrevistas orientadas a reconocer los aprendizajes.

Cuadro I - Componentes y actividades del proceso educativo: Fortalecimiento de las prácticas del uso cotidiano y conservación del agua de las comunidades El Esfuerzo, Pescador I y 2 del municipio de Turbo, Antioquia (Colombia), 2015-2016

\begin{tabular}{|c|c|}
\hline Componentes del proceso & Actividades \\
\hline $\begin{array}{l}\text { Fortalecimiento de las prácticas de uso, } \\
\text { transporte y conservación del agua }\end{array}$ & $\begin{array}{l}\text { - Encuentros iniciales: recorridos con los líderes por los barrios, } \\
\text { acompañamiento a sus prácticas de recolección y uso del agua, } \\
\text { reuniones con comunidades e instituciones. } \\
\text { - } \quad \text { Elaboración cartográfica en relación con el agua por comunidad. } \\
\text { - } \quad \text { Visita a la planta de tratamiento de agua del municipio. } \\
\text { - } \quad \text { Análisis de la calidad del agua de consumo: Toma de muestras } \\
\text { - } \quad \text { laboratorio microbiológico. } \\
\text { - } \quad \text { Actividades de multiplicación de lo aprendido en cada comunidad. }\end{array}$ \\
\hline
\end{tabular}




\section{Cuadro I-Continuacion}

\begin{tabular}{|c|c|}
\hline Componentes del proceso & Actividades \\
\hline $\begin{array}{l}\text { Promoción de prácticas de cuidado para } \\
\text { contrarrestar el desgaste corporal por el } \\
\text { transporte de agua }\end{array}$ & - Taller: Cuidando el cuerpo cuando se carga agua. \\
\hline $\begin{array}{l}\text { Promoción de hábitos para la conservación de } \\
\text { fuentes de agua }\end{array}$ & $\begin{array}{l}\text { - } \quad \text { Conversatorio sobre el cuidado de las fuentes de agua. } \\
\text { - Construcción de sillas con material reciclable. }\end{array}$ \\
\hline Divulgación de los resultados del trabajo & $\begin{array}{l}\text { - } \quad \text { Programas radiales en que los participantes narraban aprendizajes } \\
\text { - } \quad \text { experiencias. } \\
\text { - } \quad \text { Cartilla basada en la experiencia y los aprendizajes, acerca del } \\
\text { cuidado del agua. } \\
\text { - } \quad \text { Lotería educativa de prácticas y usos del agua. } \\
\text { - } \quad \text { Portafolio construido a lo largo de los talleres. }\end{array}$ \\
\hline
\end{tabular}

Participaron también 149 familias que respondieron una encuesta sobre prácticas de obtención, tratamiento, uso del agua y desgaste corporal y se vincularon a diversas actividades de multiplicación de aprendizajes en cada barrio.

\section{"El problema del agua no es solo el agua"}

Esta expresión de un funcionario de una institución de protección del ambiente concreta el sentido de los aprendizajes que queremos resaltar, pues para poner en circulación los saberes y sentires relacionados con el agua, fueron necesarios otros aspectos del espacio intersubjetivo de quienes participamos en la experiencia. De ahí que el problema del agua no es solo el agua; es también la manera como cada quien se relaciona con ella y las decisiones relacionadas con su cuidado y uso. El problema del agua es también el de reconocerse como sujetos en interacción, el de aproximarse desde diferentes historias a las maneras de aprehender el mundo y actuar en él.

\section{Diversas miradas de la misma historia}

Poner en contacto a instituciones gubernamentales y no gubernamentales, comunidades y diferentes profesionales implicó una dinámica orientada a fomentar la escucha, el respeto, la empatía, la circulación de la palabra y el cuidado de la confianza construida, a partir de la intención compartida de mejorar las condiciones del agua en la región, reconociendo de entrada la divergencia de las historias, lecturas e intereses de cada uno de los actores.

Fue así que en los encuentros de planeación y ejecución con funcionarios encargados de la protección ambiental, con quienes teníamos como objetivo realizar un taller sobre el cuidado de las fuentes de agua disponibles, partimos de escuchar la lectura de la institución sobre el proceso de poblamiento, el cual relacionaban con el deterioro del ecosistema de manglares y su planteamiento de que la única opción ante el daño ambiental era la reubicación. Partiendo entonces de esa comprensión, se explicó el sentido del proyecto y se resaltó la confianza construida con las personas. Coincidimos en que la ganancia del encuentro estaba en compartir acciones que mitiguen los daños ambientales y permitan avanzar en el acercamiento a las comunidades, lo que se reflejó en la expresión de un profesional de la institución, al afirmar que a su "saber técnico le hace falta la parte social para relacionarse con la gente" (relatoría proyecto).

En el encuentro se hacen explícitas las diferentes perspectivas sobre las condiciones de poblamiento alrededor del golfo: la institucional, que enfatizaba en la reducción del área de manglares debido al poblamiento humano y la de los pobladores, quienes ven su asentamiento en el sector como posibilidad de arraigo después del desplazamiento y su necesidad de ser reconocidos en su condición humana: "somos personas y merecemos que nos traten dignamente” (habitante Pescador 2). 
Esta sesión fue un reto, pues si bien la apuesta fundamental era el encuentro abierto entre actores relacionados con la problemática, reconocíamos la posibilidad de generar en los participantes resistencia al proceso o deterioro de la confianza. Al final, aunque cada parte se mantuvo en su perspectiva, tuvo mayor conocimiento de los argumentos de la contraparte, la complejidad de la problemática y la incertidumbre y el desacuerdo ante soluciones que incluyen a los participantes, pero no se limitan a su accionar.

Así mismo, la visita a la planta de potabilización de agua expuso diferentes visiones sobre la situación. Para algunos participantes fue una sorpresa conocer que en la planta se logra la potabilización y durante la distribución se contamina, pues "pensaba que nos mandaban el agua sucia, sin tratar" (habitante El Esfuerzo). Por su parte, desde la institución se destacaron las implicaciones de las conexiones ilegales y se resaltó que los barrios de "invasión" no pueden conectarse al servicio, lo que generó rechazo en los participantes, para quienes la ilegalidad no está en sus maneras de conseguir el agua, sino en la negación del derecho al servicio y en la exclusión selectiva del Plan de Ordenamiento Territorial, pues tienen instalación de red eléctrica, deben pagar el servicio de aseo y algunos inclusive, impuesto predial: "Nosotros también somos seres humanos, entonces si no tenemos derecho a nada allá, ¿por qué nos cobran Catastro?" (habitante Pescador 2).

$\mathrm{Al}$ invitar a los profesionales que acompañaron el proceso se realizaron reuniones previas para identificar convergencias y divergencias en las lecturas sobre la problemática, con el fin de plantear acciones pertinentes. Algunos profesionales con gran experiencia en trabajo con comunidades lograban armonizar fácilmente su propuesta conceptual y metodológica con lo construido en el proceso. Con otros, fue necesario ampliar el acompañamiento mediante recorridos adicionales en los cuales los pobladores les presentaban sus territorios, con el fin de situar el saber profesional en la realidad concreta. Así mismo, el diseño de la cartilla y las loterías, que se contrataron por convocatoria pública como requisito del ente financiador, implicó que el equipo de diseño visitara los barrios y conociera el entorno para que los participantes se vieran reflejados en las ilustraciones propuestas.
Además, en las relaciones establecidas entre actores, se expusieron diversas miradas de la misma historia, y se hicieron presentes las violencias vividas en Colombia y los derechos de las personas a la reparación de las pérdidas de la guerra, al agua y a una vida digna, lo que implicó reconocer inconsistencias estructurales que sobrepasan las posibilidades de las partes, las visiones e intereses contrarios, las incertidumbres que han acompañado las historias de las familias, lo que lleva, en últimas, a reconocer que cuando se ponen en común las diferentes posturas se puede reconocer mejor al otro aunque no siempre se llegue a acuerdos o soluciones.

\section{Verse en los otros}

La época electoral en Colombia, en 2015, cobró importancia para la dinámica del proyecto porque algunos participantes eran líderes comunitarios interesados en hacer parte de las instituciones del gobierno municipal. Esta situación afectó su participación en el proyecto, pues la adscripción a diferentes partidos políticos hizo que abandonaran el proceso. Este entorno creó también desconfianza en algunos habitantes de los barrios durante las acciones casa a casa e implicó hacer cambios en los cronogramas para no generar tensiones entre nuestras actividades y las electorales. Una participante del barrio Pescador 1 expresó:

“... un vecino me preguntó (sobre las acciones del proyecto): ¿eso es política?, yo le dije: esto no es política, estamos haciendo el esfuerzo para que el barrio salga adelante, un proyecto de aguas vivas". (habitante Pescador 1).

Los participantes además reconocieron los retos en cuanto a sus dinámicas comunitarias, pues aunque habitan espacios territoriales que tienen en común sus condiciones de vida, expresaban en las primeras reuniones: "la desunión nos tiene muertos", "vivimos aquí pero no sabemos lo que somos" (habitantes Pescador 2). Durante los encuentros reconocieron que su interacción les permitió ampliar su mirada, verse reflejados en sus compañeros de otros barrios y ver posibilidades de trabajar en una causa común: 
"He aprendido, por ejemplo, a compartir con otras personas que nunca había tenido la experiencia de compartir, con los otros dos sectores, el Esfuerzo y El Pescador 2. Mire que, aunque somos diferentes barrios, tenemos algo en común que es el agua que realmente nos hace falta a todos los tres barrios" (habitante Pescador 1).

Se valoró como significativo el reconocimiento de otros con diferentes experiencias, como lo expresó un participante del barrio El Pescador 1: "aprendimos a vernos con los otros" aunque "no es fácil trabajar en comunidad", pues "cada uno venimos con genes distintos"(habitante El Esfuerzo). Ver a otros, los cercanos y los de otros barrios, evidencia la heterogeneidad en que se dan los procesos educativos y se construyen las historias de los territorios.

Por lo anterior, el diálogo permitió a los participantes reconocer su pertenencia a una comunidad que incluye a otros con quienes comparten una necesidad común: la carencia de agua, encontrar allí espacios para la solidaridad evidenciando diferencias en las maneras como se asumen los retos cotidianos frente a una misma problemática y entorno territorial.

\section{El agua como pretexto para el diálogo}

Comprender la vida en medio de la carencia de agua implica reconocer su importancia como necesidad básica de subsistencia y su relación con aspectos culturales, sociales, políticos, ambientales y geográficos que tienen una huella importante en la historia personal y comunitaria. Para nuestro caso, esto nos lleva a analizar las condiciones en que se dio el diálogo que permitió esta experiencia.

En esta reflexión acogemos diversos elementos del diálogo a partir de este proceso educativo entre los que se incluyen: el encuentro entre extranjeros alrededor de una agenda común, la construcción de maneras particulares y pertinentes para actuar, que no necesariamente responden a modelos de educación para la salud y la importancia del conflicto como movilizador de lenguajes y develador de posturas.

Para iniciar, consideramos fundamental analizar las dinámicas y lenguajes de los participantes del proceso. Las profesionales mediadoras del proyecto partimos de lecturas de contexto, ejercicios comprensivos, diagnósticos participativos e inmersiones de campo que nos posibilitaron plantear acciones pertinentes, pero era claro que al no hacer parte del territorio, actuamos en condición de inmigrantes y, por tanto, había algunos códigos y símbolos sociales que nos eran ajenos. Algunos profesionales que se integraron al proceso desde sus áreas de experticia pertenecen al territorio y aun así desconocían la problemática o el microcontexto en que su saber se requería, priorizando la transmisión de información sobre las habilidades comunicativas o de concertación. Las comunidades se constituyeron con personas que migraron de sus lugares de origen y construyeron su arraigo como extranjeros en un territorio donde tramitan a diario sus problemáticas.

Encontrarnos desde esas diferentes condiciones de ser extranjeros nos obligó a establecer códigos comunes, a transitar en lenguajes nuevos, a construir un espacio en el que pudiéramos validar nuestra participación. Turbo fue el entorno geográfico, la carencia de agua, la razón del encuentro y la mediación del diálogo en la experiencia educativa, la que permitió ampliar la visión binaria del saber experto dialogando con el saber cotidiano, los profesionales con las comunidades, el saber hegemónico con el subalterno o el teórico con el experiencial. Esto nos permitió entrar a la interacción dialógica como sujetos, lo que implicó difuminar la frontera entre "ellos" y "nosotros", aprendiendo a construir un nosotros en el que los protagonismos fluctuaban de acuerdo a la necesidad de cada encuentro y a las maneras como fluía el diálogo. Fue una construcción heterogénea en la que todos dialogamos desde nuestro ser, historias, saberes cotidianos, expertos, experienciales, etc., y en la que no era posible homogeneizar ni a las personas de las comunidades ni a los profesionales.

Las diferencias geográficas, históricas, institucionales y disciplinarias de los participantes nos permiten analizar la potencia que puede tener el sentirse extranjero en toda acción educativa, como lo refieren Frigerio y Diker (2010, p. 8) cuando afirman que educar es disponerse a la posibilidad de una experiencia que "se gesta en el encuentro con el otro extranjero, con lo extranjero de nosotros mismos, con el pensamiento 
del otro" y que incluye pero trasciende la procedencia de cualquier tipo y se manifiesta en la acción discursiva, en una interacción que, según Serra (2003), moviliza las identidades y lecturas de la propia experiencia, pues al explicarse, el sujeto se construye. Esto permite hacer de cada proceso educativo una práctica de resignificación de todos los participantes, pues este encuentro de extranjeros complejiza la interacción e implica un esfuerzo para la comprensión mutua y del propio lugar en el mundo para afirmarlo, cuestionarlo o transformarlo.

Quizá nuestra labor como educadoras para la salud sea la de propiciar espacios de expresión para los discursos cotidianos en los que afloren "emociones, convicciones, saberes, intereses, sin que podamos prever ni su aparición, ni su secuencia, ni su intensidad" (Mariño, Cendales, 2004, p. 56), se comprenda quién es ese otro, cuál es su postura y logremos un diálogo que amplíe la posibilidad comprensiva de las problemáticas y permita actuar desde los límites y las posibilidades de las personas, los contextos y las necesidades.

En este proceso no fue una decisión inicial el no comprometernos con algún modelo concreto de educación para la salud, pero desde el lugar de reflexividad propuesto en este texto, consideramos que hacerlo así fue un buen ejercicio de colocación. Nombrar lo que queríamos proyectar en este contexto y hacer visible a cada participante en torno a su relación con el agua como causa común posibilitó el diálogo, que visto así implica una relación de conocimiento, planteada por Zemmelman (2012, p. 82) como la capacidad de cada individuo de colocarse en su mundo y como posibilidad de comprensión de los "múltiples significados que puede tener ese mundo" cuando uno se encuentra con las historias de otros, se reconoce su existencia y se valida. Más que encajar los proyectos en modelos teóricos o en experiencias de otros contextos, es importante partir de un acto epistémico que permita construir un discurso situado, que incluya elementos teorizados o experimentados por otros, pero se recree una nueva experiencia de acuerdo con los participantes.

Reconocer el lugar desde donde cada quien construye su discurso contribuye a aportar a la transformación de las personas que se propone en los procesos educativos, incluidos los de EpS, no con la pretensión de ser otros al finalizar los procesos, sino de tener más verdad, de permitirse la vulnerabilidad que implica exponerse como sujeto ante otros, de crecer en la relación de conocimiento "y esto hay que hacerlo micrológicamente, es decir, no es un discurso de grandes transformaciones, sino que empieza con una pequeña común unidad" (Rivas, 2005), problematizando la lógica de cada discurso y buscando lenguajes y necesidades comunes para el encuentro: el agua, el manglar, la vivienda, la dignidad, la vida.

Es necesario reconocer que las prácticas se dan en escenarios adversos, con intereses opuestos que hacen más complejo resolver situaciones de inequidad que se han consolidado por largo tiempo; en medio de esto, valoramos la presencia de posturas opuestas, divergencias, conflictos que, aunque generan tensiones, son estas diferencias las que permiten ser visibles para otros, pues como afirma Zuleta (2004, p. 88): "una sociedad mejor es una sociedad capaz de tener mejores conflictos. (...) de vivir no a pesar de ellos, sino productiva e inteligentemente en ellos" ${ }^{(26)}$.

Finalmente, la participación en procesos de EpS nos llama a reconocer cómo nos hemos nutrido de diversas propuestas pedagógicas, que cohabitan en nosotros y que no tienen que ser un marco restrictivo para empezar un proceso como el presentado. Nuestra labor como educadoras para la salud nos invita a aceptar la convivencia con diversas lógicas y a situarnos en los escenarios particulares desde los que cada quien entra al diálogo, para ampliar la comprensión de las problemáticas, tender puentes de relación que aporten a ese espacio complejo y heterogéneo de la experiencia humana, y a partir de allí continuar nuevos diálogos que promuevan prácticas solidarias en nuestros entornos.

\section{Referencias}

\section{ALCALDÍA MUNICIPAL DE TURBO. Plan de} desarrollo 2012-2015. Turbo, retomando el camino del progreso. Turbo: Alcaldía Municipal, 2012. Disponível em: <https://repositoriocdim.esap.edu.co/ handle/123456789/15738. Acceso em: 21 dez. 2021.

ALCALDÍA MUNICIPAL DE TURBO. Plan

de desarrollo 2016-2019. Turbo educado 
y en paz. Turbo: Alcaldía Municipal; Concejo de Turbo, 2016. Disponível em: $<$ https://turboantioquia.micolombiadigital. gov.co/sites/turboantioquia/content/ files/oooo61/3043_o1plan-de-desarrollomunicipio-de-turbo-2016--2019.pdf>. Acesso em: 9 dez. 2021.

ASTAIZA, N. et al. Diálogo intercultural en salud: una estrategia para rescatar los saberes y prácticas médicas en torno a la salud maternoinfantil de las comunidades afrocaucanas. Revista Virtual Universidad Católica del Norte, Medellín, n. 37, p. 18o-211, 2012. Disponível em: <https://www.redalyc.org/articulo. oa?id=194224568010>. Acceso em: 9 dez. 2021.

BASTIDAS, M. et al. El diálogo de saberes como posición humana frente al otro: referente ontológico y pedagógico en la educación para la salud. Investigación y Educación en Enfermería, Medellín, v. 27, n. 1, p. 104-111, 2009.

BRICEÑO, R. Siete tesis sobre la educación sanitaria para la participación comunitaria. Cadernos Saúde Pública, Rio de Janeiro, v. 1, n. 12, p. 7-30, 1996. DOI: 10.1590/So102311X1996000100002

COLOMBIA. Congreso de Colombia. Ley 1883 del 24 de enero de 2018. Por medio de la cual se otorga la categoría de Distrito Portuario, Logístico, Industrial y Comercial a Turbo. Antioquia. Diario Oficial, Bogotá, DC: 24 ene. 2018.

ESPINOSA, L.; YSUNZA, A. Diálogo de saberes médicos y tradicionales en el contexto de la interculturalidad en salud. Ciencia Ergo Sum, Toluca, v. 16, n. 3, p. 293-301, 2009. Disponível em: <https://dialnet.unirioja.es/servlet/ articulo?codigo=5035081>. Acceso em: $12 \mathrm{dez} .2021$.

FREIRE, P. Pedagogía del oprimido. México: Siglo XXI, 1975.

FRIGERIO, G.; DIKER, G. Educar: Saberes alterados. Paraná: Fundación La Hendija, 2010. GOBERNACIÓN DE ANTIOQUIA. Plan de desarrollo 2012-2015: Antioquia, la más educada. Medellín: Gobernación de Antioquia, 14 jun. 2012.
Disponível em: <https://colaboracion.dnp.gov.co/ CDT/Desarrollo\%2oTerritorial/Portal\%20 Territorial/KIT-OT/Plan-de-DesarrolloAntioquia-2012-2015.pdf >. Acceso em: 9 dez. 2017.

GRANDA, E. El sujeto la ética y la salud. In:

GRANDA, E. La saludy la vida. Quito: Ministerio de Salud Pública del Ecuador y otros, 2009. p. 97-110. 1 v.

HERNÁNDEZ, E. et al. Diálogo de saberes: propuesta para identificar, comprender y abordar temas críticos de la salud de la población. Revista Salud Uninorte, Barranquilla, v. 2, n. 33, p. 242-251, 2017. Disponível em: <http://www.scielo.org.co/ pdf/sun/v33n2/2011-7531-sun-33-02-00242.pdf>. Acesso em: 9 dez. 2021.

MARIÑO, G. El diálogo en la educación popular: entre idealizaciones y prácticas concretas. In: CENDALES, L.; MEJÍA, M.; MUÑOZ, J. Pedagogías y metodologías de la educación popular. "Se hace camino al andar”. Bogotá: Desde Abajo, 2016.

MARIÑO, G.; CENDALES, L. Educación no formal y educación popular. Hacia una pedagogía del diálogo intercultural. Caracas: Federación Internacional de Fe y Alegría, 2004. p. 54-62.

ORTEGA, P.; TORRES, A. Lola Cendales González, entre trayectos y proyectos en la educación popular. Revista Colombiana de Educación, Bogotá, n. 61, p. 333-357, 2011.

RAMASCO, M. Promoción de la salud y educación para la salud. In: PALMAR, A. Métodos educativos en salud. Barcelona: Elsevier, 2014. p. 1-19.

RESTREPO, D. La salud pública como escenario para el diálogo de saberes. Revista CES Salud Pública, Medellín, v. 1, n. 3, p. 1-3, 2012.

DOI: $10.21615 / 2133$

RIVAS, J. Pedagogía de la dignidad de estar siendo. Entrevista con Hugo Zemelman y Estela Quintar. Revista Interamericana de Educación de Adultos, Pátzcuaro, n. 1, v. 27, 2005. Disponível em: <https://www.redalyc.org/articulo. oa?id=457545085021>. Acceso em: 9 dez. 2021. RODRÍGUEZ, N.; RESTREPO, S.; ZAMBRANO, I. Carencia de agua y sus implicaciones en las prácticas alimentarias, en Turbo, Antioquia. Revista Salud Pública, Bogotá, v. 15, n. 3, p. 421-433, 2013. 
SERRA, S. Extranjeros, propios y ajenos en el hacer pedagógico. In: FRIGERIO, G.; DIKER, G. (comp.). Educación y Alteridad. Las figuras del extranjero. Textos multidisciplinarios. Buenos Aires: Novedades Educativas, 2003, p. 137-143.

\section{SERRANO, M. Educación para la salud} y participación comunitaria. Madrid: Díaz de Santos, 1990.

SERRANO, M. La educación para la salud del siglo XXI: Comunicación y salud. Madrid: Díaz de Santos, 2002.
VALADEZ, I.; VILLASEÑOR, M.; ALFARO, N. Educación para la salud: la importancia del concepto. Revista Educación y Desarrollo, Guadalajara, v. 1, n. 1, p. 43-48, 2004. Disponível em: <https://www.cucs.udg.mx/revistas/edu desarrollo/anteriores/1/oo1_Red_Valadez.pdf $>$. Acesso em: 9 dez. 2021.

ZEMMELMAN, H. El conocimiento como desafío posible. México: Instituto Politécnico Nacional, 2012. (Colección Conversaciones didácticas).

ZULETA, E. Elogio de la dificultad y otros ensayos. Cali: Fundación Estanislao Zuleta, 2004.

\section{Contribución de los autores}

Díaz-Mosquera dirigió la reflexión que dio origen al texto, realizó la escritura preliminar, la búsqueda bibliográfica y la escritura de nuevas versiones del texto. Rodríguez-Villamil participó en la reflexión que dio origen al texto, en la revisión crítica de las versiones preliminares y final, y en la escritura de aspectos de la versión final. Valencia-González participó en las reflexiones preliminares, en la búsqueda bibliográfica y en la revisión crítica de las versiones preliminares y final.

Recibido: 23/08/2019

Re-presentado: 17/09/2020; 16/07/2021

Aprobado: 20/10/2021 